\title{
Morfometría y Vascularización de la Glándula Tiroides en Conejo (Oryctolagus cuniculus)
}

\author{
Morphometry and Vascularization of the Thyroid Glands in Rabbits (Oryctolagus cuniculus)
}

\author{
Stephanie Cardoso da Silva ${ }^{1}$; Anieli Vidal Stocco ${ }^{2}$; Carlos Augusto dos Santos-Sousa ${ }^{3}$; Thais Mattos Estruc ${ }^{1}$; \\ Luiza Eula Marques ${ }^{4}$; Paulo Souza Junior ${ }^{4} \&$ Marcelo Abidu-Figueiredo $^{1}$
}

SILVA, S. C.; STOCCO, A. V.; SANTOS-SOUSA, C. A.; ESTRUC, T. M.; MARQUES, L. E.; SOUZA JUNIOR, P. \& ABIDUFIGUEIREDO, M. Morfometría y vascularización de la glándula tiroides en conejo (Oryctolagus cuniculus). Int. J. Morphol., 37(4):14041408, 2019.

RESUMEN: Las glándulas tiroides consisten en dos partes denominadas lobos que se encuentran en ambos lados de la laringe. El objetivo de este estudio fue caracterizar las medidas, topografía y vascularización de la glándula tiroides de conejos Nueva Zelanda. Las disecciones anatómicas se realizaron en 36 cadáveres adultos, 17 machos y 19 hembras, con masa corporal media de 2,5 kg y longitud cara-sacral media de $40 \mathrm{~cm}$. Los cadáveres fueron obtenidos del sector de necropsia de la Universidad. Los especímenes tuvieron la arteria aorta torácica canalada, por la cual se inyectó solución de formaldehído al $10 \%$, seguida de látex coloreado. En los machos, el lobo izquierdo de la glándula tiroidea midió 1,40 x 0,40 x 0,01 cm y el derecho 1,36 x 0,56 x 0,01 cm; en las hembras, el lobo izquierdo midió 1,50 × 0,49 × $0,01 \mathrm{~cm}$ y el derecho 1,37 × $0,48 \times 0,01 \mathrm{~cm}$. No hubo diferencia significativa $(\mathrm{p}>0,05)$ entre las medias de las medidas entre machos y hembras, pero la extensión del lóbulo izquierdo de las hembras y la anchura del lóbulo derecho de los machos fueran significativamente mayores $(\mathrm{p}<0,05)$. La extremidad craneal de los lobos tiroideos se encontraba al nivel del cartílago cricoide en casi todos los especímenes. El extremo caudal presentó topografía más variable, desde el tercer hasta el décimo anillo traqueal, habiendo predominado al nivel del quinto anillo. Invariablemente, la irrigación arterial fue determinada por las arterias tiroideas izquierda y derecha, originadas de la arteria carótida común. Las anastomosis entre las arterias tiroideas fueron comunes. La constancia en las dimensiones y irrigación de la glándula tiroidea puede ser ventajosa en la elección del Coelho como modelo experimental para el estudio de esta glándula.

PALABRAS CLAVE: Vasos sanguineos; Mediciones; Variabilidad; Conejo.

\section{INTRODUCCIÓN}

El sistema endocrino es formado por numerosas glándulas que se distribuyen por todo el cuerpo, que producen y secretan hormonas para regular muchos procesos fisiológicos.

El los mamíferos, la glándula tiroides es una de las mayores y más importante glándula endocrina. Las hormonas de tiroides regulan el crecimiento y desenvolvimiento, la actividad metabólica y la sensibilidad para otras sustancias biológicamente activas (Nussey \& Whitehea, 2001; Garcia-Reyero, 2018).

La glándula tiroides es formada por dos porciones llamadas de lóbulos que se encuentran a ambos los lados de la laringe. Los lóbulos pueden conectarse por una banda estrecha llamada de istmo, dependiendo de la especie animal (König et al., 2016).
Los avances en investigación biomédica en las últimas décadas están directamente relacionados con la utilización de animales como modelos experimentales, pues estos, posibilitan controlar y registrar las numerosas variables que no pueden ser obtenidas con los seres humanos. Además de fornecer informaciones para la sanidad animal y mejorías de gestión (Fagundes \& Taha, 2004).

Se han utilizados conejos como prototipo experimental en varios estudios, tales como: efectos de la exposición crónica al frio en la función de las glándulas tiroides (Mustafa \& Elgazzar, 2014), reconstrucción de las heridas quirúrgicas de la hemilaringectomia ampliada (Brandariz et al., 2005) y autotransplante experimental de las glándulas tiroides (Yüce et al., 2015).

\footnotetext{
${ }^{1}$ Departamento de Anatomia Animal e Humana, Universidade Federal Rural do Rio de Janeiro, Seropédica, Rio de Janeiro, Brasil.

${ }^{2}$ Hospital Veterinário, Universidade Estadual do Norte Fluminense Darcy Ribeiro, Avenida Alberto Lamego, Campos dos Goytacazes, Rio de Janeiro, Brasil.

${ }^{3}$ Laboratório de Anatomia Animal, Universidade Federal do Acre, Rodovia BR-364, Rio Branco, AC, Brasil

${ }^{4}$ Laboratório de Anatomia Animal, Universidade Federal do Pampa, Rodovia BR-472, Uruguaiana, RS, Brasil.
} 
Además, ellos han sido utilizados para estudios de toxicología (Zablotny et al., 2012), farmacología (Idiz et al., 2016) y quirúrgica (Szabo at al., 2016). Sin embargo, a pesar de ser ampliamente utilizados en la investigación, algunos aspectos de su macro anatomía necesitan de una descripción más detallada, ante todo el sistema vascular arterial de las glándulas endocrinas.

El objetivo de esta investigación fue caracterizar las medidas, topografía y vascularización de las glándulas tiroides en conejos de ambos los sexos.

\section{MATERIAL Y MÉTODO}

El comité de ética en investigación de la Universidad Federal Rural do Rio de Janeiro aprobó esta investigación (número 23083.002379 / 2007-08).

Se utilizaron 36 cadáveres frescos de conejos adultos (Oryctolagus cuniculus) de la raza Nueva Zelanda, 17 machos y 19 hembras, de peso medio y extensión rostro-sacra de $2,5 \mathrm{~kg}$ y $40 \mathrm{~cm}$, respetivamente. Los animales fueron obtenidos de la unidad de necropsia de la universidad. Los cadáveres fueron puestos en decúbito lateral derecho y por incisión torácica se retiraron las costillas $6^{\mathrm{a}}$ y $7^{\mathrm{a}}$. Su aorta torácica fue diseccionada y canulada para lavarse el sistema arterial con suero fisiológico y fueron fijadas con solución de formaldehido a $10 \%$. Subsecuentemente, el sistema vascular fue rellenado con la coloración azul de la Petrolax S-65 (Refinería Duque de Caxias - REDUC / Petrobrás, Duque de Caxias, Rio de Janeiro, Brasil). Luego, cada cadáver fue sumergido en una caja de polietileno de baja densidad con capacidad de 500 litros conteniendo solución de formaldehido a $10 \%$ durante 5 días para completar el proceso de fijación y polimerización del látex. Después de este periodo los cadáveres fueron lavados en agua corriente y disecados. Se realizó una incisión en la piel, y los músculos ventrales del pescuezo fueron disecados para la exposición de la tráquea, glándulas tiroides y su vascularización. Las glándulas tiroides fueron disecadas in situ para la caracterización de la topografía. Fueron obtenidas las medidas de las glándulas tiroides con un parquímetro digital: extensión (longitud entre el polo craneal y el polo caudal), anchura (longitud entre margen ventral y margen dorsal) y espesura (obtenido por la medición del tercio medio del lóbulo tiroideo, en las caras medial y lateral). El origen de las arterias tiroides también fue determinado. Todo el material diseccionado fue fotografiado usándose una camera de modelo Nikon Coolpix@ L820.

Los dados obtenidos fueron documentados y presentados en tablas y la terminología adoptada se basaba en el International Committe on Veterinary Gross Anatomical Nomenclature (2017).

Análisis estadístico. La desviación media y la desviación padrón de las medidas de la glándula tiroides han sido calculadas y comparadas en ambos antimeros y sexos por el teste t no pareado. Para comprobar si las distribuciones de frecuencia observada para los 30 animales estaban de acuerdo con la literatura, el teste Qui-quadrado se realizó para testar la hipótesis de nulidad de la esqueletopia de los lóbulos derecho e izquierdo de la tiroides. La relación entre las medidas tiroideas y la extensión rostro-sacra fue calculada por el coeficiente de correlación " $r$ " variando de -1 y +1 , o sea, -1 $\leq \mathrm{r} \leq+1$. Un valor de $\mathrm{p}<0,05$ fue considerado estadísticamente significativo.

\section{RESULTADOS Y DISCUSIÓN}

Los resultados de las medidas de las glándulas tiroides están representados en las Tablas I, II y III. La frecuencia y

Tabla I. Valores de las mediciones $(\mathrm{cm})$ de la glándula tiroidea en las hembras.

\begin{tabular}{lccc}
\hline Hembras & $\begin{array}{c}\text { Lóbulo } \\
\text { derecho }\end{array}$ & Lóbulo izquierdo & Valor de P \\
\hline Extensión & $1,37 \pm 0,20$ & $1,51 \pm 0,20$ & $0,04^{*}$ \\
Anchura & $0,48 \pm 0,12$ & $0,49 \pm 0,10$ & 0,73 \\
Espesura & $0,01 \pm 0,01$ & $0,01 \pm 0,008$ & 0,23 \\
\hline
\end{tabular}

*diferencia significativa $(\mathrm{p}<0,05)$.

Tabla II. Valores de las mediciones (cm) de la glándula tiroidea en machos.

\begin{tabular}{lccc}
\hline \multicolumn{1}{c}{ Machos } & $\begin{array}{c}\text { Lóbulo } \\
\text { derecho }\end{array}$ & $\begin{array}{l}\text { Lóbulo } \\
\text { izquierdo }\end{array}$ & Valor de P \\
\hline Extensión & $1,36 \pm 0,18$ & $1,40 \pm 0,33$ & 0,71 \\
Anchura & $0,56 \pm 0,14$ & $0,49 \pm 0,09$ & 0,08 \\
Espesura & $0,01 \pm 0,007$ & $0,01 \pm 0,01$ & 0,66 \\
\hline
\end{tabular}

\begin{tabular}{llcc}
\hline \multicolumn{1}{c}{ Mediciones } & \multicolumn{1}{c}{ Machos } & Hembras & Valor de P \\
\hline Extensión del lóbulo derecho & $1,36 \pm 0,18$ & $1,37 \pm 0,20$ & 0,85 \\
Extensión del lóbulo izquierdo & $1,40 \pm 0,33$ & $1,51 \pm 0,20$ & 0,23 \\
Anchura del lóbulo de recho & $0,56 \pm 0,14$ & $0,48 \pm 0,12$ & 0,07 \\
Anchura del lóbulo izquierdo & $0,49 \pm 0,09$ & $0,49 \pm 0,10$ & 0,90 \\
Espesura del lóbulo derecho & $0,01 \pm 0,007$ & $0,01 \pm 0,01$ & $0,02 *$ \\
Espesura del lóbulo izquierdo & $0,01 \pm 0,01$ & $0,01 \pm 0,008$ & 0,38 \\
\hline
\end{tabular}

Tabla III. Comparación de valores de las mediciones $(\mathrm{cm})$ de la glándula tiroidea en machos y hembras. *diferencia significativa $(\mathrm{p}<0,05)$ 
el porcentual simples de la relación de cada glándula tiroidea como los anillos traqueales han sido demostrados en las Tablas IV y V.

Hubo correlación lineal positiva entre la extensión rostro-sacra con la anchura de lo lobo derecho en los ma-
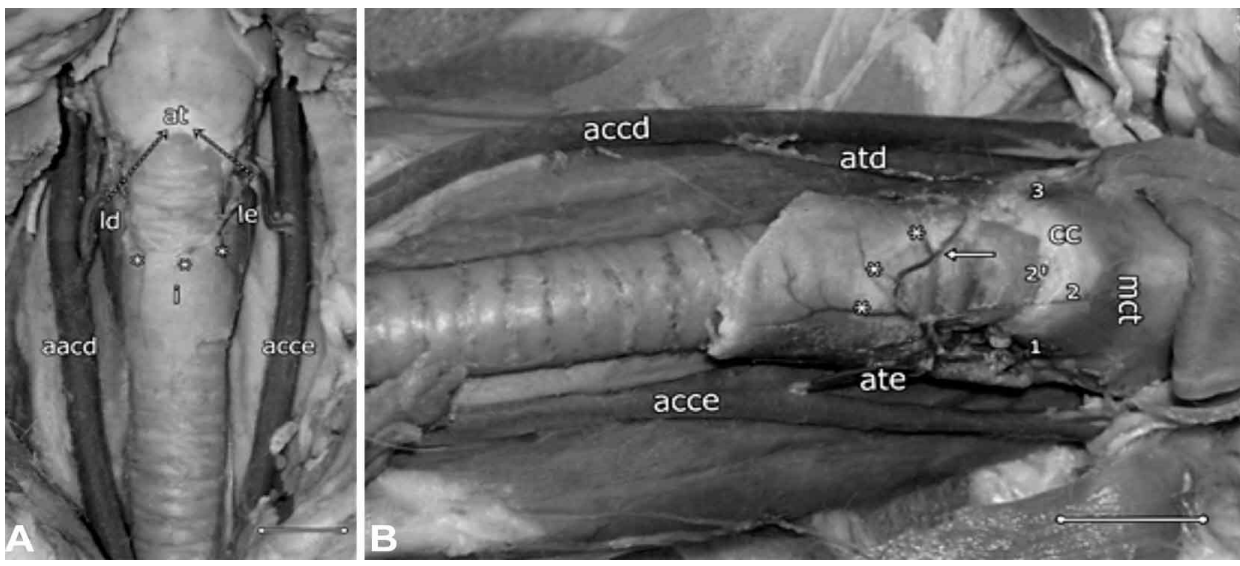

Fig. 1. Fotomacrografia de la vascularización, morfología y localización de la glándula tiroides en conejos de Nueva Zelanda. En A, visión ventral: accd, arteria carótida común derecha; acce, arteria carótida común izquierda; Id, lobo derecho de la glándula tiroides; le, lobo izquierdo de la glándula tiroides; at, arterias tiroideas; $(*)$, ramas de la arteria tiroidea; i, istimo. En B, visión ventro-lateral izquierda: acce, arteria carótida común izquierda; accd, arteria carótida común derecha; ate, arteria tiroidea izquierda; atd, arteria tiroidea derecha; (*) ramas para el lobo izquierdo e istmo; (*), rama de comunicación (anastomosis); 1, 2, 2’y 3, ramas para el cartílago cricoides (cc) y para el musculo cricotiroideo (mct). Barra de escala: $10 \mathrm{~mm}$.

Tabla IV. Frecuencia absoluta y porcentaje simple de la topografía de la glándula tiroides en hembras de conejos Nueva Zelanda.

\begin{tabular}{cccc}
\hline Glándula tiroides & Nivel & Lóbulo derecho & Lóbulo izquierdo \\
\hline \multirow{3}{*}{ Polo cranial } & Cartílago cric oides & $18(94,7 \%)$ & $18(94,7 \%)$ \\
& $1^{\circ}$ anillo traqueal & $1(5,3 \%)$ & $1(5,3 \%)$ \\
& $3^{\circ}$ anillo traqueal & $2(10,5 \%)$ & - \\
& $4^{\circ}$ anillo traqueal & $4(21,1 \%)$ & $2(10,5 \%)$ \\
& $5^{\circ}$ anillo traqueal & $7(36,8 \%)$ & $9(47,6 \%)$ \\
Polo caudal & $6^{\circ}$ anillo traqueal & $2(10,5 \%)$ & $3(15,8 \%)$ \\
& $7^{\circ}$ anillo traqueal & $3(15,8 \%)$ & $1(5,3 \%)$ \\
& $8^{\circ}$ anillo traqueal & $1(5,3 \%)$ & $1(5,3 \%)$ \\
& $9^{\circ}$ anillo traqueal & - & $2(10,5 \%)$ \\
\hline
\end{tabular}

Tabla V. Frecuencia absoluta y porcentaje simple de la topografía de la glándula tiroides en machos y conejos Nueva Zelanda.

\begin{tabular}{cccc}
\hline Glándula tiroides & Nivel & Lóbulo derecho & Lóbulo izquierdo \\
\hline \multirow{3}{*}{ Polo cranial } & Cartílago cricoides & $16(94,1 \%)$ & $16(94,1 \%)$ \\
& $1^{\circ}$ anillo traqueal & $1(5,9 \%)$ & $1(5,9 \%)$ \\
& $3^{\circ}$ anillo traqueal & $1(5,9 \%)$ & - \\
& $4^{\circ}$ anillo traqueal & $4(23,4 \%)$ & $2(11,8 \%)$ \\
& $5^{\circ}$ anillo traqueal & $2(11,8 \%)$ & $5(29,4)$ \\
Polo caudal & $6^{\circ}$ anillo traqueal & $5(29,4 \%)$ & $5(29,4)$ \\
& $7^{\circ}$ anillo traqueal & $2(11,8 \%)$ & $3(17,6 \%)$ \\
& $8^{\circ}$ anillo traqueal & $1(5,9 \%)$ & $1(5,9 \%)$ \\
& $9^{\circ}$ anillo traqueal & $1(5,9 \%)$ & $1(5,9 \%)$ \\
& $10^{\circ}$ anillo traqueal & $1(5,9 \%)$ & - \\
\hline
\end{tabular}

Todavía, datos que incluyen la morfología de esta estructura no han sido relatados. En este caso, considerando el conejo de Nueva Zelanda como ejemplo experimental de amplia utilización, carece de informaciones a respecto de su morfología, morfometria, topografía y vascularización que pueden ser útiles en programas de formación y desarrollo de nuevas técnicas para el estudio de esta glándula.

En el presente investigación se observó que las glándulas tiroides del conejo Nueva Zelanda presentó dos lóbulos ordenados lateralmente a la tráquea unidos por un istmo ventralmente, semejante a los observados en el ratón (Yamasaki, 1990), conejo blanco japonés (Yamasaki, 
1996) y en los mamíferos domésticos (König et al.), con excepción del chancho que posee un tejido glandular compactado en la cara ventral de la tráquea, a los cuales su punto craneal se posiciona en el cartílago tiroideo, mientras el extremo caudal del punto alcanza la abertura torácica (König et al.).

Yamasaki (2016) al estudiar distintas especies de marsupiales diprotodones observo que en relación a la topografía de la glándula tiroidea, siempre estuvo localizada ventrolateralmente entre laringe y tráquea. Sin embargo, raramente se observó la presencia del istmo como se ha informado anteriormente en su estudio con marsupiales poliproptodones (Yamasaki, 1993) y musaraños asiáticos (Yamasaki, 1997).

En estudio con 60 espécimen (30 machos y 30 hembras) de Cavia cobaya, Yamasaki (1994) observó la presencia de largos lóbulos tiroideos elipsoides lateralmente a la tráquea, considerándose de aspectos morfológicos muy uniformes. Sin embargo, la presencia del istmo se observó solo en 6 espécimen machos y 7 espécimen hembras.

Comparando machos y hembras no hube diferencia en las medidas de los lóbulos de la glándula tiroides, con excepción en la espesura de la glándula tiroidea derecha que es mayor en las hembras $(p=0,0198)$. Analizando los machos, no se observó diferencia entre las medidas del lobo derecho e izquierdo de la tiroides. En las hembras el lobo derecho de la tiroides presento extensión menor que el lobo izquierdo $(\mathrm{p}=0,0472)$. En ovejas de la raza Santa Inés no se observó ninguna diferencia en las disposiciones de las glándulas Lima et al. (2009a), así como en el perro doméstico (Rodrigues et al., 2016). Mientras Novo et al. (2009) al evaluar la glándula tiroides del perro a través del examen ultrasonografico observaron mayores medidas de extensión y largura del lobo izquierdo en relación al derecho, sin, sin embargo, separaren las pruebas por sexo.

En fetos de bovinos con sangre europea, Santos et al. (2008) observaron diferencias entre los antimeros izquierdo y derecho, para las mediciones de anchura, con valores mayores para el izquierdo, y diferencias entre machos y hembras únicamente para el antimeros izquierdo relativo a la extensión de la glándula tiroidea, en la cual, los mayores valores fueron obtenidos en los machos.

La glándula tiroidea en el conejo se encuentra ventrolateralmente en la región de la tráquea, semejante al observado en los perros (Rodrigues et al.) y al gato morisco (Carvalho et al., 2003). En esta investigación, tanto en machos como en hembras, los lóbulos derecho e izquierdo comenzaron predominantemente a nivel del cartílago cricoides. En las hembras el final vario de $3^{\circ}$ a $9^{\circ}$ anillo traqueal, mientras que en los machos $3^{\circ}$ a $11^{\circ}$ anillo traqueal, parcialmente similar a los valores obtenidos por Rodrigues et al. en perros domésticos.

En todos los conejos disecados, el suministro arterial de la glándula tiroides en ambos los antimeros vino de la arteria tiroidea, una rama de la arteria carótida común. Después de su origen, la arteria tiroidea emitió ramas para el musculo cricotireoide y para la parte craneal de los correspondientes lóbulos e istmo. Estos hallazgos se parecen a los observados por Yamasaki (1997) en musarañas asiáticas, en lo cual, no se observó arterias tiroideas caudales. Sin embargo, el origen de la arteria tiroides craneal en su estudio se originó constantemente de la arteria carótida externa.

La presencia de anastomosis entre las arterias tiroideas derecha e izquierda fue observada en el presente estudio, aunque no se ha registrado su frecuencia. Kulcsar et al. (2008) en estudio retrospectivo de tiroidectomías en pacientes humanos de un hospital universitario durante el periodo de 2000 a 2003, observaron que las tiroidectomías parciales representaron 22,9 $\%$ de los procedimientos. En este contexto, la presencia de la anastomosis entre las arterias tiroideas derecha e izquierda debe ser considerada en las excusiones de los procedimientos quirúrgicos que buscan minimizar las hemorragias que dificulten la visualización del campo quirúrgico y, en consecuencia, dificultan la extirpación parcial de la glándula.

La glándula tiroidea de algunas especies es irrigada por las arterias tiroideas craneales y caudales, como observado en perros (Rodrigues et al.), gato morisco (Carvalho et al.), rumiantes (Santos et al.; Lima et al., 2009a,b).

De acuerdo con Yamasaki (1990) en estudio realizado con ratas se observó que las arterias tiroideas craneales y caudales estuvieron presentes en la vascularización de la glándula tiroidea, sin embargo, estas se originaron en $76 \%$ de las veces en los machos y $72 \%$ de las veces en las hembras a partir de la arteria carótida externa.

Se observaron diferencias significativas en la cuantidad de arterias tiroideas por Yamasaki (1994) en estudio con 60 espécimen de chancho-de-la-india, el cual observo la presencia de hasta 5 tipos de arterias. Sin embargo, el mismo, señaló que la arteria tiroidea craneal fue la más frecuente, siendo un poco más común en los machos que en las hembras. En nuestro estudio la arteria tiroidea fue única en todos los especímenes. Entonces, aunque ella presentara una inserción mayor en la extremidad craneal de los lóbulos de la glándula tiroidea, la adición dela expresión craneal no sería adecuado debido a la eminente ausencia de una arteria tiroidea caudal.

A constancia observada en el suministro de la glándula tiroides por una sola arteria tiroidea en ambos los sexos y antimeros de conejos de Nueva Zelanda observada en nues- 
tro estudio, sugiere que estos animales pueden ser un buen modelo experimental para el desarrollo de técnicas que involucren la glándula tiroidea. Además de aportar informaciones al campo de la anatomía animal comparada.

AGRADECIMIENTOS. El presente trabajo fue realizado con apoyo de la Coordinación de Perfeccionamiento de Personal de Nivel Superior - Brasil (CAPES) - Código de Financiamiento 001; FAPERJ e CNPq.

\section{SILVA, S. C.; STOCCO, A.V.; SANTOS-SOUSA, C. A.; ESTRUC, T. M.; MARQUES, L. E.; SOUZA JUNIOR, P. \& ABIDU- FIGUEIREDO, M. Morphometry and vascularization of the thyroid glands in rabbits (Oryctolagus cuniculus). Int. J. Morphol., 37(4):1404-1408, 2019.}

SUMMARY: The thyroid glands consist of two parts called lobes located on both sides of the larynx. The aim of this study was to characterize the measures, topography and arterial irrigation of New Zealand's rabbits thyroid glands. The anatomical dissections were performed in 36 adult cadavers, 17 males and 19 females, with a mean body mass of $2.5 \mathrm{~kg}$ and a mean cranium-sacral length of $40 \mathrm{~cm}$. The specimens were obtained from the necropsy unit of the University. The cadavers had the thoracic aorta artery cannulated, through which $10 \%$ formaldehyde solution was injected, followed by injection colored latex. In males, the left lobe of the thyroid gland measured $1.40 \times 0.40 \times 0.01 \mathrm{~cm}$ and the right $1.36 \times 0.56 \times 0.01 \mathrm{~cm}$; in females, the left lobe measured $1.50 \times 0.49 \times 0.01 \mathrm{~cm}$ and the right one was $1.37 \times 0.48 \times 0.01 \mathrm{~cm}$. There was no significant difference $(\mathrm{p}>0.05)$ between the means of the measurements between males and females, but the left lobe length of the females and the right lobe width of the males were significantly higher ( $p<0.05)$. The cranial extremity of the thyroid lobes was at the level of the cricoid cartilage in almost all specimens. The caudal end showed more variable topography, from the third to the tenth tracheal ring in few specimens, but predominated at the level of the fifth ring. Invariably, arterial irrigation was determined by the left and right thyroid arteries, originating from the common carotid artery. Anastomoses between the thyroid arteries were common. The constancy in dimensions and irrigation of the thyroid gland may be advantageous in choosing the rabbit as an experimental model for the study of this gland.

Rabbit.

KEY WORDS: Blood vessels; Measurements; Variability;

\section{REFERENCIAS BIBLIOGRÁFICAS}

Brandariz, J. A. \& Grau, M. Tracheal wall for the reconstruction of extended hemilaryngectomy in the rabbit model. Otolaryngol. Head Neck Surg., 132(5):770-5, 2005.

Carvalho, S. F. M.; Santos, A. L. Q.; Andrade, M. B.; Magalhães, L. M.; Ribeiro, F. M.; Cruz, G. C. \& Malta, T. S. Morfometria e vascularização arterial da glândula tireóide do gato mourisco, Herpailurus yagouaroundi (Severtzow, 1858) felidae. ARS Vet., 19(3):216-8, 2003.

Fagundes, D. J. \& Taha, M. O. Modelo animal de doença: critérios de escolha e espécies de animais de uso corrente. Acta Cir. Bras., 19(1):59-65, 2004.
Garcia-Reyero, N. The clandestine organs of the endocrine system. Gen. Comp. Endocrinol., 257:264-71, 2018.

Idiz, U. O.; Aysan, E.; Can, I.; Buyukpinarbasili, N.; Yardimci, E. Y. \& Bektasoglu, $H$. The effects of lauromacrogol on thyroid tissue in rabbits. Is this a safe option for the treatment of nodular thyroid disease? Ann. Ital. Chir., 87:1927, 2016.

König, H. E. \& Liebich, H. G. Anatomia dos Animais Domésticos: Texto e Atlas Colorido. $6^{\text {th }}$ ed. Porto Alegre, Artmed, 2016.

Kulcsar, M. A. V.; Friguglietti, C. U. M.; Cividanes, R. \& Brandão, L. G. Análise retrospectiva das tireoidectomias realizadas por residentes de cirurgia geral em hospital universitário. Rev. Bras. Cir. Cabeça Pescoço, 37(2):67-70, 2008.

Lima, E. M. M.; Ferreira, P. M.; Silva, L. R.; Vianna, A. R. C. B.; Santana, M. I. S.; Silva, F. O. C. \& Severino, R. S. Morfometria e suprimento arterial da glândula tireóide em ovinos da raça Santa Inês. Vet. Not., 15(1):35-40, 2009a.

Lima, E. M. M.; Santana, M. I. S.; Silva, F. O. C.; Severino, R. S.; Vianna, A. R. C. B. \& Nemer, E. M. T. Suprimento arterial sanguíneo para a glândula tireóide em caprinos da raça Saanen. Vet. Not., 15(2):95-101, 2009 b.

Mustafa, S. \& Elgazzar, A. Influence of chronic exposure to cold environment on thyroid gland function in rabbits. Horm. Metab. Res., 46(8):546-9, 2014.

Novo, A. C. M. P.; de Carvalho, C. B. \& Alves, R. B. M. Ultrassonografia das glândulas tireóideas em cães (Canis familiaris, Linnaeus, 1758). J. Bras. Cienc. Anim., 2(3):135-49, 2009.

Nussey, S. \& Whitehea, S. Endocrinology. An Integrated Approach. Oxford, BIOS Scientific Publishers, 2001.

Rodrigues, A. B. F.; Costa, N. Q; de Aguiar, R. R.; Di Filippo, P. A. \& de Almeida, A. J. Análise morfológica, topográfica e vascularização da glándula tireóide em cães (Canis familiaris). Rev. Bras. Med. Vet., 38(3):316-22, 2016.

Santos, A. L. Q.; Neto, A. M.; Moura, L. R.; Pereira, H. C. \& Silva Júnior, L. M. Vascularização arterial, forma, topografia e morfometria da glândula tireóide em fetos de bovinos com sangue europeu. Vet. Not., 14(1):63-70, 2008.

Szabo, Z.; Bradley, K. \& Cahalane, A. K. Rabbit soft tissue surgery. Vet. Clin. North Am. Exot. Anim. Pract., 19(1):159-88, 2016.

Yamasaki, M. Comparative anatomical studies of thyroid and thymic arteries: I. Rat (Rattus norvegicus albinus). Am. J. Anat., 188(3):249-59, 1990.

Yamasaki, M. Comparative anatomical studies on the thyroid and thymic arteries. II. Polyprotodont marsupials. J. Anat., 183(Pt. 2):359-66, 1993.

Yamasaki, M. Comparative anatomical studies on the thyroid and thymic arteries. IV. Rabbit (Oryctolagus cuniculus). J. Anat., 188(Pt. 3):557-64, 1996.

Yamasaki, M. Comparative anatomical studies on the thyroid and thymic arteries. V. House musk shrew (Suncus murinus). Okajimas Folia Anat. Jpn., 73(6):293-300, 1997.

Yamasaki, M. Comparative anatomical studies on the thyroid and thymic arteries. VI. Diprotodont marsupials. Anat. Sci. Int., 91(3):258-73, 2016.

Yüce, I.; Okuducu, H.; Çaglı, S.; Vural, A.; Gündogdu, R.; Abdülrezzak, Ü.; Arlı, T.; Aydın, M. \& Güney, E. Experimental autotransplantation and cryopreservation of the thyroid gland. Head Neck, 37(7):940-5, 2015.

Zablotny, C. L.; Rasoulpour, R. J.; Pitt, J. A. \& Carney, E. W. Assessment of the developmental and reproductive toxicity of diiodomethyl-p-tolylsulfone in rats and rabbits. Regul. Toxicol. Pharmacol., 62(3):474-81, 2012.

Dirección para correspondencia:

Prof. Dr. Marcelo Abidu-Figueiredo

Departamento de Anatomía Animal e Humana

Universidade Federal Rural do Rio de Janeiro.

Rodovia BR-465, km 07, CEP 23890-000

Seropédica

Río Janeiro - BRASIL

Email: marceloabidu@gmail.com

Recibido : 05-05-2019

Aceptado: 02-07-2019 